\title{
Commentary
}

\section{Multiple System Atrophy}

\section{Clues from Inclusions}

\author{
Rudy Castellani, M.D. \\ From the Division of Neuropathology, Department of Pathology, \\ University of Maryland, Baltimore, Maryland
}

Multiple system atrophy (MSA) was added to the list of neurodegenerative diseases defined by cytoplasmic inclusions when Papp et al ${ }^{1}$ demonstrated specific oligodendroglial inclusions, referred to as glial cytoplasmic inclusions (GCls), in brains of patients affected by MSA. Their observation has since allowed more accurate interpretation of the pathological differential diagnosis of parkinsonian, cerebellar, and autonomic disorders and has further supported the concept of MSA as an entity. Moreover, MSA appears unique among neurodegenerative diseases in that its hallmark inclusion affects oligodendrocytes, suggesting that glial changes and glial-neuronal interactions may be early events in neurodegeneration, and emphasizing the potential importance of myelin metabolism in disease pathogenesis. In the current issue of this journal, Matsuo and colleagues ${ }^{2}$ expand on this finding by providing evidence that myelin degeneration in patients with MSA is more widespread than previously recognized.

\section{Development of the Multiple System Atrophy Concept: Historical Perspective}

MSA is a sporadic neurodegenerative disease encompassing three previously described clinical syndromesstriatonigral degeneration (SND), olivopontocerebellar atrophy (OPCA), and Shy-Drager syndrome (SDS) - with a common pathology. ${ }^{3}$ The current view of MSA, however, took a number of years to unfold and is probably best understood from a historical perspective.

In 1900, Déjerine and Thomas introduced the term olivopontocerebellar atrophy (OPCA) when they described two middle-aged patients with an ataxic disorder and pathological changes in the brainstem and cerebellum. The striatum and substantia nigra were not examined, or at least not commented on. ${ }^{4}$ Déjerine and Thomas, and later Loew ${ }^{5}$ under the tutelage of Déjerine himself, recognized OPCA cases as "atypical" when they had a hereditary component or, interestingly, when there were associated CNS lesions, but their concept of "atypical OPCA" fell quickly into disuse. ${ }^{6}$ Nevertheless, OPCA as described by Déjerine and Thomas persisted as an entity and was the subject of a number of studies in the middle part of the 20th century ${ }^{7,8}$ despite the fact that the distinction between familial and sporadic OPCA (see below) and the frequency of associated lesions were not fully appreciated.

In 1960, Shy and Drager described a neurological syndrome with autonomic failure and parkinsonism, SDS, ${ }^{9}$ in which striking orthostatic hypotension was the common clinical feature. Previously described cases of orthostatic hypotension, in retrospect, may have represented SDS, ${ }^{10-12}$ but these were not as well characterized. Shy and Drager noted degeneration of the intermediolateral cell columns of the spinal cord (later recognized as the anatomical substrate of autonomic failure), in addition to neuronal loss and/or gliosis at many other sites including the caudate nuclei, substantia nigra, cerebellar Purkinje's cells, and inferior olivary nuclei. Lewy bodies were noted in a minority of the early SDS cases, raising the possibility of "transitional" forms between SDS and Parkinson's disease, ${ }^{13}$ although it should be noted that examination of the striatum and (now) the presence of GCls will usually permit distinction between these two apparently separate processes. It is also important to remember that the description of SDS (as well as SND) preceded the availability of levodopa for diagnosis and treatment of parkinsonism. ${ }^{14}$

The year before, van der Eecken et al had presented "Striopallidal-nigral degeneration: An hitherto undescribed lesion in paralysis agitans" to the American Association of Neuropathologists. Their account was published in 1960 and then described in more detail in 1961 and 1964 under the designation "striatonigral degeneration" (SND). ${ }^{15-17}$ SND patients typically exhibited parkinsonism and the major foci of neuronal loss were in the striatum and the substantia nigra. Lewy bodies were not

\section{Accepted for publication July 20, 1998}

Address reprint requests to Rudy Castellani, M.D., Division of Neuropathology, Department of Pathology, University of Maryland, Baltimore, 22 South Greene St., Baltimore, MD 21201. 
a constant feature in SND, being present in the substantia nigra in only one of four cases. OPCA was also described in one case but, as in SDS, only in retrospect was OPCA found to be an important component of the underlying disease. It also seems surprising that striatal pathology in patients with parkinsonism was not described until the early 1960s. In fact, pathological accounts of typical SND were described earlier, but were included under the label of Parkinson's disease. ${ }^{18}$ Given that approximately $10 \%$ of patients with parkinsonism have MSA, it is indeed likely that many such cases "have swelled the ranks of Parkinson's disease clinics"14 unrecognized as a separate disease.

Finally, in a 1969 paper titled "Orthostatic Hypotension and Nicotine sensitivity in a Case of Multiple System Atrophy," Graham and Oppenheimer reported the case of a 60-year-old man who presented with clinical features of SDS as well as cerebellar ataxia exacerbated by cigarette smoking. ${ }^{19}$ Progressive clinical deterioration ensued and he died about 2 1/2 years later. Neuropathological examination disclosed degeneration of the striatum, substantia nigra, pons, inferior olivary nucleus, cerebellar Purkinje's cells, and intermediolateral cell column of the spinal cord. From careful examination of this single case and review of pertinent literature, Graham and Oppenheimer made the observation that SDS, SND, and OPCA often co-existed clinically and pathologically and were likely different expressions of one underlying disease. For better or worse, they introduced the term "multiple system atrophy" for this group of diseases to avoid "unnecessary confusion caused by inventing new names."

\section{Clinical Neuropathology and Patterns of Degeneration}

According to current understanding, almost all MSA patients develop parkinsonism or cerebellar signs. Thus, about $80 \%$ of patients are parkinson-predominant (SNDpredominant) and $20 \%$ are cerebellar-predominant (OPCA-predominant). ${ }^{20}$ Pure SND is relatively common clinically, whereas pure OPCA is unusual. Onset of autonomic dysfunction (SDS) in MSA is variable but is usually present at some point during the disease course. Patients with severe autonomic dysfunction tend to be younger and to have a shorter survival. ${ }^{20}$ As alluded to previously, the frequent occurrence of parkinsonism emphasizes the often difficult distinction between MSA and disorders such as Parkinson's disease and progressive supranuclear palsy (Steele-Richardson-Oslewski syndrome).

Descriptions in recent studies of patterns of pathological involvement of gray matter are generally an extension of the original accounts, noting neuronal loss and gliosis in the striatum (more in the putamen than in the caudate nucleus), substantia nigra, locus ceruleus, inferior olivary nucleus, pontine nuclei, cerebellar Purkinje's cells, intermediolateral cell column in the thoracic spinal cord, and Onuf's nucleus in the sacral spinal cord. Other populations variably affected include the external pallidum, thalamus, vestibular nuclei, dorsal motor nucleus of the va- gus, and corticospinal tracts. ${ }^{20-22}$ The degree of involvement tends to follow clinical signs, with the striatonigral system severely affected in parkinson-predominant and pure SND cases, and the olivopontocerebellar system severely affected in cerebellar-predominant cases. ${ }^{20}$ Pathological changes in the olivopontocerebellar system may be subtle or nonexistent in pure SND cases, but because pure OPCA is unusual, striatal and nigral pathology is present in the majority of MSA cases. Autonomic dysfunction in MSA is related to degeneration of the intermediolateral cell column and Onuf's nucleus. ${ }^{13}$ Dementia, on the other hand, is not a major presenting problem, although some cortical deficits emerge with psychometric testing ${ }^{23}$ and subtle pathological abnormalities affecting the cerebral cortex are described. ${ }^{24,25}$

\section{Absence of Well-Defined Genetic Etiology}

MSA is a largely sporadic condition and there have been few studies investigating potential genetic etiologies. In a recent study of 80 well-characterized MSA patients, Bandmann et $\mathrm{al}^{26}$ found no alterations in either the SCA-1 or SCA-3 gene, indicating that MSA is likely distinct from autosomal dominant ataxias associated with unstable trinucleotide repeats. ${ }^{27}$ Gotoda et al ${ }^{28}$ identified a mutation in the gene for $\alpha$-tocopherol transfer protein in one patient with adult-onset spinocerebellar dysfunction, substantiating the notion that altered vitamin $\mathrm{E}$ metabolism leads to spinocerebellar pathology; however, the patient's neurological disorder more closely resembled Friedreich's ataxia than MSA. In a case-controlled study, Nee et $\mathrm{al}^{29}$ found that patients with MSA had significantly more potential exposures to metal, dusts, fumes, plastic monomers and additives, organic solvents, and pesticides than the control population. The authors concluded that MSA develops as a result of a genetically determined selective vulnerability in the nervous system, although the nature of the selective vulnerability was not elucidated and no disease-associated genetic locus was identified. It should also be emphasized here that, aside from one report of $\mathrm{GCls}$ in SCA-1 pathology, ${ }^{30} \mathrm{GCl}$ s continue to represent a specific hallmark of sporadic MSA and are generally not a component of the inherited ataxias. Therefore, the available data indicate that MSA is an acquired condition distinct from CNS diseases with well-defined genetic causes. In this regard it is interesting to note that increased amounts of iron are consistently found in the striatum of MSA patients both neuropathologically and on neuroimaging. ${ }^{31,32} \mathrm{Fe}(\mathrm{II})$ as a potent catalyst of reactive oxygen species has been implicated in the pathogenesis of common neurodegenerative diseases. ${ }^{33,34}$ At present, evidence for oxidative stress in MSA remains circumstantial.

\section{Glial Cytoplasmic Inclusions as the Pathological Hallmark of Multiple System Atrophy: Characteristics, Distribution, and Pathogenic Implications}

In their original study in 1989, Papp et al documented the important finding of argyrophilic oligodendroglial inclu- 
sions in the brains of patients with MSA. ${ }^{1}$ The GCls were first noted by Gallyas silver impregnation, used to demonstrate Alzheimer's neurofibrillary pathology; they were localized largely to the white matter and to cells with morphological features of oligodendrocytes in all 11 cases. Neuronal inclusions with similar characteristics to GCls were also reported, ${ }^{35}$ but these are now considered insufficient in density or specificity to be a reliable feature of MSA. ${ }^{36} \mathrm{No} \mathrm{GCl}$ s were seen in any of the age-matched normal controls and GCls were absent from a variety of other neurodegenerative diseases. Subsequent studies have verified the presence of GCls in MSA as well as the conclusion of Papp et al that GCls are sensitive and specific hallmarks of MSA. ${ }^{37-42}$ The relevance of GCls, not only to characterizing a nosological entity but also in terms of enhancing neuropathological interpretation of parkinsonian, ataxic, and autonomic disorders, is selfevident.

By routine light microscopy, $\mathrm{GCls}$ are faint eosinophilic inclusions that eccentrically displace the nucleus. The Gallyas silver technique produces selective dark staining of inclusions with a clean background, making it more useful in demonstrating GCls than Bodian or Bielschowsky techniques, which have more background and less pronounced staining of the inclusion. The various silver techniques show $\mathrm{GCl}$ s are sickle-shaped to flame-shaped to ovoid, sometimes superficially resembling neurofibrillary tangles. The fact that $\mathrm{GCls}$ were not detected earlier, though somewhat surprising, may reflect their less intense staining with commonly used silver impregnations and the fact that GCls are essentially negative with other common stains, including phosphotungstic acid hematoxylin, periodic acid-Schiff, Masson trichrome, Alcian blue, thioflavine S, Congo red, oil red O, or Sudan black B. ${ }^{3,36}$ On ultrastructural examination, GCls are comprised of loosely aggregated filaments with cross-sectional diameters of 20 to $30 \mathrm{~nm}$. The filaments often entrap cytoplasmic organelles such as mitochondria and secretory vesicles, have no limiting membrane, and are reported to have tubular profiles and electrondense granules along much of their length. ${ }^{36,39}$ By immunocytochemistry, GCls are consistently positive for ubiquitin and $\alpha$-B crystallin, and less intensely positive for $\alpha$ - and $\beta$-tubulins. ${ }^{3,36}$ Ubiquitin and $\alpha$-B crystallin positivity is not surprising, as both proteins accumulate in a variety of poorly soluble inclusions as a general indicator of cellular stress. MAP5 positivity is also reported. ${ }^{38,39}$ GCls are negative for neurofilaments, glial fibrillary acidic protein, myelin basic protein, vimentin, actin, desmin, myosin, and cytokeratin. The variable literature on tau immunocytochemistry may reflect the phosphorylation state of the tau epitope, as GCls are generally negative for phosphate-dependent tau antibodies and positive for normal adult tau; ${ }^{43}$ this is in contrast to the neurofibrillary pathology of Alzheimer's disease. The apparent absence of phosphorylated tau further distinguishes GCls from glial lesions in corticobasal degeneration and progressive supranuclear palsy (eg, tufted astrocytes, coiled bodies, astrocytic plaques). ${ }^{36,43}$

The regional distribution of $\mathrm{GCls}$ within the brain is complex. Significant numbers of GCls have consistently been found in the putamen, pallidum, lateral caudate nucleus, basis pontis, spinal cord intermediate gray matter, internal capsule, and middle cerebellar peduncle, ${ }^{36}$ indicating a general tendency for GCls to accumulate in and around degenerated brain regions. Not surprisingly, some have viewed this as evidence that $\mathrm{GCl}$ formation is a secondary phenomenon. On the other hand, Papp and Lantos found from their distribution study that $\mathrm{GCls}$ preferentially involve suprasegmental motor systems, supraspinal autonomic systems, and their targets, while sparing primary sensory areas and cortical and subcortical limbic structures, suggesting a "system-bound" oligodendroglial degeneration. ${ }^{35}$ Moreover, the presence of $\mathrm{GCls}$ in such areas as motor cortex, cerebral white matter, subthalamic nucleus, and brainstem reticular formation (structures that do not typically degenerate in MSA) and their relative absence in the substantia nigra, locus ceruleus, and inferior olivary nucleus suggest that $\mathrm{GCl}$ formation cannot be attributed solely to a reaction to neuronal damage. ${ }^{35}$ Inoue et $\mathrm{al}^{40}$ also found a general relationship between the density of $\mathrm{GCls}$ and the severity of white matter tract involvement, although again GCls were more widespread than neuronal loss and the density of $\mathrm{GCls}$ in the cerebellar white matter in relation to severity of pathology was more complex. Few GCls were found in cerebellar white matter with severe OPCA, whereas numerous GCls were sometimes found with little or no OPCA, furthering the argument that GCls may be an early lesion and not necessarily a reaction to neuronal injury.

\section{The Oligodendrocyte and Myelin as Potential Primary Targets}

GCls consistently localize to cells with oligodendroglial characteristics. By immunocytochemistry, GCl-containing cells are immunoreactive with antibodies against Leu-7, carbonic anhydrase enzyme II, and transferrin. ${ }^{39,42}$ Ultrastructural characteristics of $\mathrm{GCl}$-containing cells are also consistent with oligodendroglial origin. $1,37,39,42$ As mentioned, oligodendroglial inclusions are rarely encountered in other neurodegenerative diseases; only in MSA does the oligodendrocyte represent the predominant inclusion-bearing cell. It is somewhat paradoxical that MSA rather eloquently affects selected gray matter "systems" whereas its hallmark inclusionbearing cell produces myelin. Implicating the oligodendrocyte as the primary target, therefore, means providing a mechanism for region-specific oligodendrocyte dysfunction or a mechanism whereby oligodendrocyte dysfunction leads to pronounced destruction of selected vulnerable neuronal populations. To date there is little evidence that oligodendrocytes may be subtyped according to the neuronal populations they subserve. Older classifications of Mori and LeBlond ${ }^{44}$ and Stensaas and Stensaas $^{45}$ subtyped oligodendrocytes based on fine structural features of the oligodendrocyte nucleus and processes and myelin sheaths, respectively, providing indirect evidence for functional specialization, but no conclusive evidence for tract specificity. More recently, 
oligodendrocyte development has been characterized by immunophenotype, shedding some light on the development of macroglia, but likewise has not progressed to the point of tract or functional specificity. ${ }^{46}$ Additionally, single oligodendrocytes have been shown to myelinate axons of different anatomical tracts, casting some doubt on the possibility that oligodendrocytes may be classified based on the functional properties of corresponding axons. ${ }^{47,48}$ In MSA, GCls are reported to involve so-called perivascular, perifascicular, and perineuronal oligodendrocytes without further qualification as to subtype. In essence, then, GCls involve all morphological types of oligodendrocytes with varying frequency in various anatomical regions. ${ }^{3,35}$ It therefore appears that oligodendroglial disturbance in MSA, whether or not it occurs primarily, tends to be more generalized and would likely have to occur in conjunction with intrinsic vulnerability of selected neuronal populations, the nature of which remains to be determined.

In the current issue of this journal, Matsuo and colleagues $^{2}$ document extensive myelin degeneration in MSA brains by demonstrating widespread white matter (but not GCl) immunopositivity with anti-EP antisera and monoclonal antibody QD-9. Both antibodies recognize synthetic peptide QDENPVV (corresponding to human myelin basic protein residues 82-88) and were previously shown to immunolabel degenerated myelin in multiple sclerosis and infarcted brains, but not myelin in normal brain. ${ }^{49}$ The presence of unusual myelin basic protein epitopes in MSA, in both affected and unaffected brain regions, substantiates the notion of widespread oligodendroglial dysfunction in MSA and highlights white matter disease as an integral component. Nevertheless, the question of how oligodendrocyte degeneration might lead to neuronal loss, to the extent encountered in MSA, remains. It is noteworthy in this regard that axonal damage is known to occur in otherwise classical demyelinating conditions. ${ }^{50,51}$ Furthermore, the critical trophic influences that are described between oligodendrocytes and axons $^{52}$ indicate that oligodendroglial pathology would likely affect neuronal function. Oligodendrocyte pathology in the form of (presumably chronic) inclusions, on the other hand, causing selective and severe gray matter damage, would be unusual. It is safe to conclude that despite the compelling evidence presented so far, more studies are needed before GCls or degenerated myelin epitopes may be interpreted as primary lesions in the neurodegenerative process of MSA.

\section{Inclusions: Etiology Versus Epiphenomenon}

The difficulty in establishing a direct association between inclusions and etiology is not unique to MSA. While inclusions provide empirical pathological hallmarks for a number of neurodegenerative diseases and are particularly strategic for studying disease pathogenesis, ${ }^{53,54}$ a direct association between inclusion and etiology (as opposed to epiphenomenon) remains unproven for most neurodegenerative disorders. This includes neurofibrillary pathology of Alzheimer disease and progressive supranuclear palsy, Lewy bodies of Parkinson's disease and diffuse Lewy body disease, Pick's bodies of Pick's disease, the various tau pathologies of corticobasal degeneration, and Rosenthal fibers of Alexander's disease. On the other hand, recent reports demonstrating tau gene mutation in familial multiple system tauopathy, ${ }^{55}$ and the immunolocalization of $\alpha$-synuclein, a protein mutated in chromosome 4-linked familial Parkinson's disease, ${ }^{56}$ to Lewy bodies of idiopathic Parkinson's disease, ${ }^{57}$ provide direct links between genetic lesions and abnormal gene products accumulating in hallmark inclusions. Therefore, as the biochemical composition of $\mathrm{GCls}$ and associated myelin abnormalities continue to be elucidated, rigorous study of the pathological lesions of MSA, with the hope of determining etiology and treatment strategies, appears warranted. This is particularly true in light of the lack of a genetic basis for the disease.

\section{Conclusions}

After nearly a century of study MSA is now known to be a specific, albeit heterogeneous, neurodegenerative disease encompassing olivopontocerebellar atrophy, striatonigral degeneration, and Shy-Drager syndrome. It was through thoughtful case examination, detailed neuropathological study, and advances in molecular genetics that the entity of MSA was fully realized. The relatively recent identification of unique cytoplasmic inclusions and widespread white matter abnormalities have broadened the scope of the neurodegenerative process, and have challenged traditional views of MSA as a disease primarily of gray matter. While studies on GCls and oligodendroglial dysfunction will continue to provide insight into the pathophysiology and perhaps etiology of MSA, the progress already made will continue to be an important lesson in neurodegenerative disease pathogenesis and careful clinicopathological observation.

\section{Acknowledgments}

I am grateful to Dr. Mark A. Smith for critical review of this commentary and helpful suggestions, and to Philippe Pary for valuable assistance.

\section{References}

1. Papp MI, Kahn JE, Lantos PL: Glial cytoplasmic inclusions in the CNS of patients with multiple system atrophy (striatonigral degeneration, olivopontocerebellar atrophy and Shy-Drager syndrome). J Neurol Sci 1989, 94:79-100

2. Matsuo A, Akiguchi I, Lee GC, McGeer EG, McGeer PL, Kimura J: Myelin degeneration in multiple system atrophy detected by unique antibodies. Am J Pathol 1998, 153:735-744

3. Lantos PL, Papp MI: Cellular pathology of multiple system atrophy: a review. J Neurol Neurosurg Psychiatry 1994, 57:129-133

4. Déjerine J, Thomas AA: L'atrophie olivo-ponto-cérébelleuse: Nouv Iconog Salpatriere 1900, 13:330-370

5. Loew P: L'atrophie olivo-ponto-cérébelleuse, Thesis, 1903-1904: University of Paris

6. Berciano J: Olivopontocerebellar atrophy. J Neurol Sci 1982, 53:253272 
7. Critchley M, Greenfield JC: Olivo-ponto-cerebellar atrophy. Brain 1948, 71:343-364

8. Greenfield JG: The spino-cerebellar degenerations. Blackwell Scientific Publications, Oxford, 1954

9. Shy GM, Drager GA: A neurological syndrome associated with orthostatic hypotension: a clinical-pathological study. Arch Neurol 1960, 2:511-527

10. Bradbury S, Eggleston C: Postural hypotension: a report of three cases. Am Heart J 1925, 1:73-86

11. Langston W: Orthostatic hypotension: report of a case. Ann Intern Med 1936, 10:688-695

12. Young $\mathrm{RH}$ : Association of postural hypotension with sympathetic nervous system dysfunction: case report with review of neurological features associated with postural hypotension. Ann Intern Med 1944, 15:910-916

13. Bannister R, Oppenheimer DR: Degenerative diseases of the nervous system associated with autonomic failure. Brain 1972, 95:457-474

14. Quinn N: Multiple system atrophy: the nature of the beast. J Neurol Neurosurg Psychiatry 1989, suppl:78-89

15. van der Eecken $\mathrm{H}$, Adams RD, van Bogaert L: Striopallidal-nigral degeneration. An hitherto undescribed lesion in paralysis agitans. J Neuropathol Exp Neurol 1960, 19:159-161

16. Adams RD, van Bogaert $L$, van der Eecken $H$ : Dégénérescences nigro-striées et cerebello-nigro-striées. Psychiat Neurol 1961, 142: 219-259

17. Adams RD, van Bogaert L, van der Eecken H: Striato-nigral degeneration. J Neuropathol Exp Neurol 1964, 23:584-608

18. Messing Z: Atrophie olivo-ponto-cerebelleuse dans un cas de maladie de Parkinson. Rev Neurol (Paris) 1930, 60:498-499

19. Graham JG, Oppenheimer DR: Orthostatic hypotension and nicotine sensitivity in a case of multiple system atrophy. J Neurol Neurosurg Psychiatry 1969, 32:28-34

20. Wenning GK, Ben Shlomo Y, Magalhães M, Daniel SE, Quinn NP: Clinical features and natural history of multiple system atrophy: an analysis of 100 cases. Brain 1995, 117:835-845

21. Wenning GK, Ben-Shlomo Y, Magalhães M, Daniel SE, Quinn NP: Clinicopathological study of 35 cases of multiple system atrophy. J Neurol Neurosurg Psychiatry 1995, 58:160-166

22. Wenning GK, Tison F, Ben Shlomo Y, Daniel SE, Quinn NP: Multiple system atrophy: a review of 203 pathologically proven cases. Mov Disord 1997, 12:133-147

23. Robbins TW, James M, Lange KW, Owen AM, Quinn NP, Marsden CD: Cognitive performance in multiple system atrophy. Brain 1992, 115:271-291

24. Fujita T, Doi M, Ogata T, Kanazawa I, Mizusawa H: Cerebral cortical pathology of sporadic olivopontocerebellar atrophy. J Neurol Sci 1993, 116:41-46

25. Arai N, Papp MI, Lantos PL: New observations on ubiquitinated neurons in the cerebral cortex of multiple system atrophy (MSA). Neurosci Lett 1994, 182:197-200

26. Bandmann O, Sweeney MG, Daniel SE, Wenning GK, Quinn N, Marsden CD, Wood NW: Multiple-system atrophy is genetically distinct from identified inherited causes of spinocerebellar degeneration. Neurology 1997, 49:1598-1604

27. Koeppen AH: The hereditary ataxias. J Neuropathol Exp Neurol 1998, 57:531-543

28. Gotoda T, Arita M, Arai $H$, Inoue $K$, Yokota T, Fukuo $Y$, Yazaki $Y$, Yamada N: Adult-onset spinocerebellar dysfunction caused by a mutation in the gene for the $\alpha$-tocopherol-transfer protein. N Engl J Med 1995, 333:1313-1318

29. Nee LE, Gomez MR, Dambrosia J, Bale S, Eldridge R, Polinsky RJ: Environmental-occupational risk factors and familial associations in multiple system atrophy: a preliminary investigation. Clin Auton Res 1991, 1:9-13

30. Gilman S, Sima AA, Junck L, Kluin KJ, Koeppe RA, Lohman ME, Little R: Spinocerebellar ataxia type 1 with multiple system degeneration and glial cytoplasmic inclusions. Ann Neurol 1996, 39:241-255

31. Martin WR, Roberts TE, Ye FQ, Allen PS: Increased basal ganglia iron in striatonigral degeneration: in vivo estimation with magnetic resonance. Can J Neurol Sci 1998, 25:44-47

32. Schwarz J, Weis S, Kraft E, Tatsch K, Bandmann O, Mehraein P, Vogl $T$, Oertel WH: Signal changes on MRI and increases in reactive microgliosis, astrogliosis, and iron in the putamen of two patients with multiple system atrophy. J Neurol Neurosurg Psychiatry 1996, 60:98101

33. Smith MA, Wehr K, Harris PLR, Siedlak SL, Connor JR, Perry G: Abnormal localization of iron regulatory protein in Alzheimer's disease. Brain Res 1998, 788:232-236

34. Smith MA, Harris PLR, Sayre LM, Perry G: Iron accumulation in Alzheimer disease is a source of redox-generated free radicals. Proc Natl Acad Sci USA 1997, 94:9866-9868

35. Papp MI, Lantos PL: The distribution of oligodendroglial inclusions in multiple system atrophy and its relevance to clinical symptomatology. Brain 1994, 117:235-243

36. Chin SS-M, Goldman JE: Glial inclusions in CNS degenerative diseases. J Neuropathol Exp Neurol 1996, 55:499-508

37. Yagishita S, Amano N, Iwabuchi K, Hasegawa K, Kowa K: Argyrophilic glial intracytoplasmic inclusions in multiple system atrophy: immunocytochemical and ultrastructural study. Acta Neuropathol 1992, 84:273-277

38. Arai N, Nishimura M, Oda M, Morimatsu $Y$, Ohe R, Nagatomo H: Immunohistochemical expression of microtubule-associated protein 5 (MAP5) in glial cells of multiple system atrophy. J Neurol 1992, 109:102-106

39. Arima K, Murayama S, Murayama M, Inose T: Immunocytochemical and ultrastructural studies of neuronal and oligodendroglial cytoplasmic inclusions in multiple system atrophy 1 . Neuronal cytoplasmic inclusions. Acta Neuropathol 1992, 83:453-460

40. Inoue M, Yagashita S, Ryo M, Hasegawa K, Amano M, Matsushita M: The distribution and dynamic density of oligodendroglial cytoplasmic inclusions (GCls) in multiple system atrophy: a correlation between the density of $\mathrm{GCls}$ and the degree of involvement of striatonigral degeneration and olivopontocerebellar systems. Acta Neuropathol 1997, 93:585-591

41. Kato S, Nakamura H, Hirano A, Ito H, Llena JF, Yen S-H: Argyrophilic ubiquitinated inclusions of Leu-7-positive glial cells in olivopontocerebellar atrophy (multiple system atrophy). Acta Neuropathol 1991, 82:488-493

42. Murayama S, Arima K, Nakazato Y, Satoh J, Oda M, Inose T: Immunocytochemical and ultrastructural studies of neuronal and oligodendroglial cytoplasmic inclusions in multiple system atrophy 2. Oligodendroglial inclusions. Acta Neuropathol 1992, 84:32-38

43. Cairns NJ, Atkinson PF, Hanger DP, Anderton BH, Daniel DE, Lantos PL: Tau protein in the glial cytoplasmic inclusions of multiple system atrophy can be distinguished from abnormal tau in Alzheimer's disease. Neurosci Lett 1997, 230:49-52

44. Mori S, Leblond CP: Electron microscopic identification of three classes of oligodendrocytes and a preliminary study of their proliferative activity in the corpus callosum of young rats. J Comp Neurol 1970, 139:1-30

45. Stensaas LJ, Stensaas SS: Astrocytic neuroglial cells, oligodendrocytes and microgliacytes in the spinal cord of the toad. I. Light microscopy. Z Zellforsch 1968, 84:473-489

46. Ludwin SK: The pathobiology of oligodendrocytes., J Neuropathol Exp Neurol 1997, 56:111-124

47. Bleichenki PV, Celio MR: Relationship between oligodendrocytes and axons. Neuroreport 1997, 8:3965-3967

48. Sternberger NH, Itoyama $\mathrm{Y}$, Kies MW, Webster H deF: Immunocytochemical method to identify basic protein in myelin-forming oligodendrocytes of newborn rat C.N.S. J Neurocytol 1978, 7:251-263

49. Matsuo A, Lee GC, Terai K, Takami K, Hickey WF, McGeer EG, McGeer PL: Unmasking of an unusual myelin basic protein epitope during the process of myelin degeneration in humans: a potential mechanism for the generation of autoantigens. Am J Pathol 1997, 150:1253-1266

50. Griffin JW, Li CY, Ho TW, Tian M, Gao CY, Zue P, Mishu B, Cornblath DR, Macko C, McKhann GM, Asbury AK: Pathology of motor-sensory axonal Guillain-Barre syndrome. Ann Neurol 1996, 39:17-28

51. Trapp BD, Peterson J, Ransohoff RM, Rudick R, Mork S, Bo L: Axonal transection in the lesions of multiple sclerosis. N Engl J Med 1998, 338:278-285

52. Kaplan MR, Meyer-Franke A, Lambert S, Bennett V, Duncan ID, Levinson SR, Barres BA: Induction of sodium channel clustering by oligodendrocytes. Nature 1997, 386:724-728

53. Castellani R, Perry G, Harris PLR, Cohen M, Sayre L, Salomon RG, Smith MA: Advanced lipid peroxidation end products in Alexander's disease. Brain Res 1998, 787:15-18 
54. Castellani R, Smith MA, Richey P, Perry G: Glycoxidation and oxidative stress in Parkinson disease and diffuse lewy body disease. Brain Res 1996, 737:195-200

55. Spillantini MG, Crowther RA, Jakes R, Hasegawa M, Goedert M: $\alpha$-synuclein in filamentous inclusions of lewy bodies from Parkinson's disease and dementia with lewy bodies. Proc Natl Acad Sci USA 1998, 95:6469-6473

56. Polymeropoulos MH, Lavedan C, Leroy E, Ide SE, Dehejia A, Dutra A,
Pike B, Root H, Rubenstein J, Boyer R, Stenroos ES, Chandrasekharappa S, Athanassiadou A, Papapetropoulos T, Johnson WG, Lazzarini AM, Duvoisin RC, Di lorio G, Golbe LI, Nussbaum RL: Mutation in the $\alpha$-synuclein gene identified in families with Parkinson's disease. Science 1997, 276:2045-2047

57. Spillantini MG, Murrell JR, Goedert M, Farlow MR, Klug A, Ghetti B: Mutation in the tau gene in familial multiple system tauopathy with presenile dementia. Proc Natl Acad Sci USA 1998, 95:7737-7741 